\title{
The Role of Flexible Bronchoscope in the Diagnosis of the Pulmonary Tracheobronchial Tuberculosis in Children-Report of Four Cases and Review of Literature
}

\author{
Linhua Shu ${ }^{1 *}$, Hassan Muhammad ${ }^{2}$, Liying Han ${ }^{3}$, Qin Xiong ${ }^{1}$, Yijing Hu ${ }^{1}$, Huiyan Zhang ${ }^{1}$ and Dan Wang ${ }^{1}$ \\ ${ }^{1}$ Department of Pediatrics Pulmonology, Shanghai Children's Hospital, Shanghai Jiao Tong University, Shanghai 200062, China \\ ${ }^{2}$ Department of Pediatric Orthopedics, Huashan Hospital, Fudan University, Shanghai 200040, China \\ ${ }^{3}$ Department of Pediatrics, Shengjing Hospital of China Medical University, Shenyang 110004, China
}

"Corresponding author: Linhua Shu, Department of Pediatrics Pulmonology, Shanghai Children's Hospital, Shanghai Jiao Tong University, Shanghai 200062, China, Tel: + 201102265; E-mail: shulinhua@126.com

Received date: July 10, 2014, Accepted date: March 30, 2015, Published date: April 07, 2015

Copyright: $\odot 2015$ Shu L, et al. This is an open-access article distributed under the terms of the Creative Commons Attribution License, which permits unrestricted use, distribution, and reproduction in any medium, provided the original author and source are credited.

\begin{abstract}
The diagnosis of tracheobronchial tuberculosis (TBTB) on the grounds of clinical and radiological findings in children is more difficult due to the lack of etiological confirmation. We aimed to elucidate the application of flexible bronchoscopy (FB) in the diagnosis of tuberculosis (TB) with involvement of tracheobronchial wall named TBTB. Pathological changes typically include mucosal and submucosal edema and hyperemia. It also comprise of tubercular-like nodules, caseous necrosis and fibrous hyperplasia. Acid-fast bacilli staining is done from tissue slices or bronchial alveolar lavage fluid (BALF) sediment and a positive stain confirms the diagnosis. It also aids in differential diagnosis of complicated and refractory pneumonia. Furthermore, it can be beneficial in gastric washing and sputum collection. Flexible bronchoscopy can be performed for pediatric purposes efficiently and effectively. It is a safe and easy procedure.
\end{abstract}

Keywords: Flexible bronchoscope; Tracheobronchial tuberculosis; Pneumonia; Bronchial alveolar lavage fluid; Acid-fast Bacilli staining

\section{Introduction}

Pulmonary tuberculosis (TB) is one of the major health problems globally [1]. The incidence of pediatric pulmonary TB is increasing unexpectedly at an alarming rate in Northeast part of China. TB in childhood is often more difficult to diagnose due to the atypical clinical and radiological features and the difficulty to expectorate [2]. Standard protocols and guidelines set by the World Health Organization (WHO) [3], for the management of TB in children, have been widely followed in our country. However, in children, after the birth till the age of 15 years old, it often goes undiagnosed. The difficulty in isolating the pathogenic organism from the respiratory specimens, especially sputum samples with the limitations of mycobacterial culture and negative tuberculin skin test are some of the causes for unsuccessful diagnosis of TB in young children.

Tracheobronchial tuberculosis (TBTB) is a special type of pulmonary TB. The tracheobronchial tuberculose focuses often injure the tracheobronchial wall and lead to a series of pathological changes [4]. As reported in previous studies, about $10-40 \%$ of patients with acute pulmonary TB had TBTB [5]. TBTB can arise as the primary manifestation of TB or during the course of the disease. It is often misdiagnosed as pneumonia, bronchial asthma, or foreign body aspiration [6].

The diagnosis of TBTB is easily delayed or mistaken because of non-specific clinical manifestations and the low incidence of positive acid-fast bacilli staining [7]. The diagnosis based on radiological evidence, were frequently incorrect and hence could not replace or support the etiological confirmation. In some individuals, compatible mixed infections maybe co-exist in the same lesion of the lung [8]. It is difficult to differentiate from pneumonia based on clinical features, tuberculin skin test and chest radiography. For a few complicated cases with extrapulmonary manifestations, such as anemia, malnutrition, lymphadenopathy, hepatomegaly and/or splenomegaly, meningeal signs, and/or ascites, it was confused with refractory pneumonia due to atypical manifestations and lack of pathogenic identification.

WHO recommends etiological confirmation of pulmonary tuberculosis by the detection of acid-fast bacilli in respiratory specimens [9]. However in young children, gastric lavage is recommended for the collection of respiratory secretions. Difficulties arise when the smear examinations from sputum or gastric lavage are negative. Henceforth, more aggressive procedures need to be undertaken in these patients in order to establish the diagnosis.

A number of studies confirm the usefulness of flexible bronchoscopy in the diagnosis of pulmonary tuberculosis [10-12]. Flexible bronchoscopy with bronchial aspiration and bronchial alveolar lavage fluid (BALF) under local anesthesia is a relatively safe procedure and well tolerated by most patients. Complications are known but rare in occurrence.

The following 4 cases showed the importance of flexible bronchoscopy in the etiological confirmation from BALF in children who were treated as refractory pneumonia for a long time.

\section{Case 1}

A nine years old boy experienced repeated cough for five years. Five years ago, he suffered from cold repeatedly, and also suffered from pneumonia for every one or two months. He developed hepatomegaly, splenomegaly, lymphadenopathy (in jowl, axilla, groin), and moderate 
Citation: Shu L, Muhammad H, Han L, Xiong Q, Hu Y, et al. (2015) The Role of Flexible Bronchoscope in the Diagnosis of the Pulmonary Tracheobronchial Tuberculosis in Children-Report of Four Cases and Review of Literature. J Bacteriol Parasitol 6: 223. doi: $10.4172 / 2155-9597.1000223$

Page 2 of 7

anemia before four and half years. He suffered from a severe pulmonary infection with abundant pleural effusion and ascites about three years ago. Two years ago, the size of his spleen was about $20 \mathrm{~cm}$ $\times 12 \mathrm{~cm} \times 6 \mathrm{~cm}$, and he underwent lienectomy due to severe anemia. After the operation, he was very vulnerable and so suffered from cold frequently and also had tympanitis with hearing weakness and often diarrhea. Skin was easily infected and was cankered due to mosquito bites.

He was weak (hypodynamia and fatigue), motionless, and fleshless. His mother had been diagnosed as a case of pulmonary tuberculosis, but the child had never been diagnosed as pulmonary tuberculosis. Physical examination after admission revealed the following: $\mathrm{T}$ : $37.0^{\circ} \mathrm{C}$, P: 90 beats/min, RR: 26 times/min, BP: 84/52 mmHg, BW: 26 kgs. Clinical features of fleshless and sedimentation of chicken pox on the skin were seen. Scar approximately $15 \mathrm{~cm}$ long can be seen on the left side of abdomen. No enlargement of lymph nodes in jowl, axilla, and groin. Liver was $2 \mathrm{~cm}$ under right lowest rib and $3 \mathrm{~cm}$ under xiphoid process of sternum. No abnormalities of the heart and lungs were found by auscultation. Valuable accessory examinations were done during the time of illness. Chest X-ray films done on nine occasions in the past five years revealed patchy consolidation in the right middle lobe. The chest CT scan taken three years ago demonstrated that the presence of partial consolidation in the right lung accompanied with narrowing in the middle lobular bronchus without cavity formation, calcification and pleural involvement. Three years ago, the ultrasound reports demonstrated the enlargement of lymph nodes in the bilateral jowl, neck, supraclavicular fossa, axilla and groin. The peripheral blood routine test illustrated that the $\mathrm{WBC}$, $\mathrm{RBC}$ and platelet were decreased, and accompanied with moderate anemia. After the lienectomy, RBC and platelet returned to normal, but WBC soared abruptly. C-reactive protein (CRP) values were normal during seven occasions. Immunoglobulin test and sub group of $\mathrm{T}$ lymphocytes were at the normal levels. Hemolytic anemia and anemia due to malnutrition were excluded. Lymphoma was also excluded after two bone marrow punctures. Lienectomy pathology reports revealed hypersplenism and lymph nodes hyperplasia both at mesentery and splenic hilum. On the day of admission, chest enhancement scanning demonstrated presence of multiple large consolidations in the inferior lingual segment of the left lung and also in the posterior segment of right upper lobe and apical segment of right lower lobe with air-bronchogram and bronchiectasis in it. Furthermore, presence of bronchial stenosis was detected in the left lingual segment (Figure 1). Cardiac and abdominal ultrasound examinations revealed normal findings except for the absence of spleen. Pulmonary ventilation function and exhaled nitric oxide were found to be in the normal range. The lymph node biopsy on left groin reported lymphatic reactive hyperplasia. Tuberculin skin tests (purified protein derivative, PPD) were done on ten different occasions in the past five years and the report turned out negative in all the cases. Similarly, serum tuberculosis antibody test was performed five times out of which, in only one incident it turned out positive and the rest of it were negative. Polymerase chain reaction (PCR) tests performed to diagnose tuberculosis were also negative.

Induced sputum smears were taken three times for detection of acid-fast bacilli but were found to be absent. $\mathrm{KOH}$ stain for presence of fungi was also done and it was reported negative.

Serum tuberculosis antibody (colloidal gold immunization) and tuberculin skin test (purified protein derivative, PPD) were found to be negative.

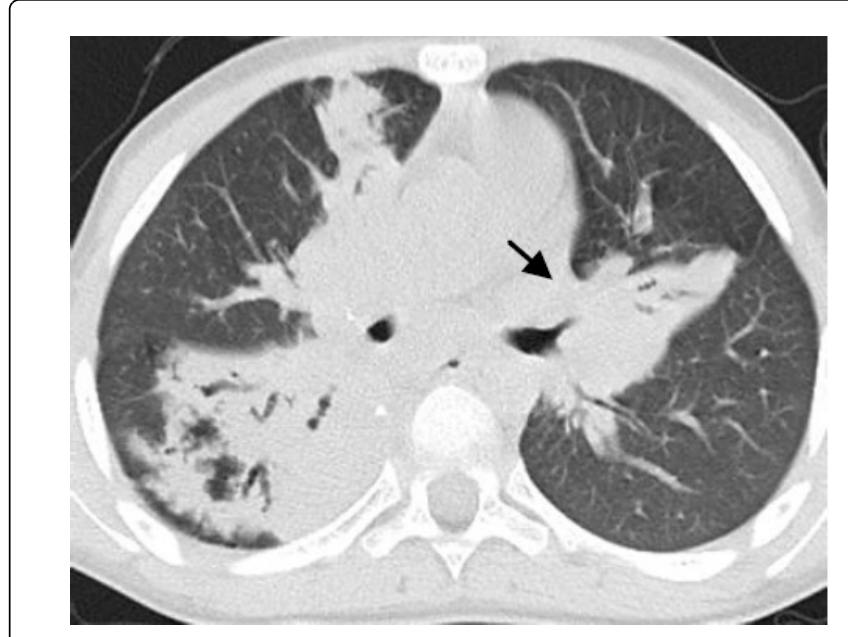

Figure 1: Computer tomography of a nine years old boy with recurrent pneumonia for 5 years showed multiple large consolidations in inferior lingual segment on the left lung and posterior segment of right upper lobe and apical segment of right lower lobe with air-bronchogram and bronchiectasia in it. There was bronchial stenosis at left lingual segment (see arrow).

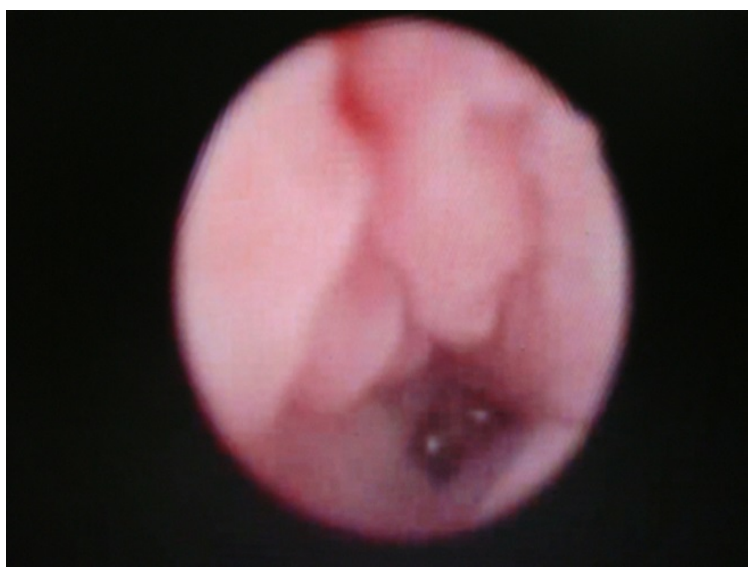

Figure 2: Bronchoscopic appearances seen commonly in endobronchial tuberculosis (EBTB) with narrowing of right inferior lobar bronchus. Bronchial mucosa is edema and hyperplasia. The mucosa of trachea and bronchi was protruded and sunken all around the internal wall. The endobronchial mucosa looked scraggly like cobblestone with pink colour.

After receiving consent from his parents, flexible bronchoscopy (Penyax FB-10V, $\Phi: 3.6 \mathrm{~mm}$ ) was performed under general anesthesia. During the operation, the mucosa of trachea and bronchi was protruded and depressed all around the internal wall (Figure 2). The cartilaginous ring of trachea had disappeared. The trachea had become narrow. BALF obtained from both the right and left lungs were centrifuged and the sedimentation was smeared immediately for ordinary Gram stain, acid-fast stain and $\mathrm{KOH}$ stain. The final results confirmed the presence of acid-fast bacilli in BALF from both the right and left lung. Endobronchial tuberculosis (EBTB) was diagnosed based on bacteriological identification by flexible bronchoscopy. Anti- 
Citation: Shu L, Muhammad H, Han L, Xiong Q, Hu Y, et al. (2015) The Role of Flexible Bronchoscope in the Diagnosis of the Pulmonary Tracheobronchial Tuberculosis in Children-Report of Four Cases and Review of Literature. J Bacteriol Parasitol 6: 223. doi: $10.4172 / 2155-9597.1000223$

Page 3 of 7

tuberculosis treatment was initiated immediately. One month later, the condition of the boy improved compared to his previous state. The cough was relieved and hearing had improved. He was energetic and had a good appetite and absence of diarrhea. At 1 and 2 months follow-up flexible bronchoscopy, the endobronchial mucosa was seen to be smoother than before and the smear of BALF for acid-fast bacilli was negative consecutively.

Under bronchoscopy, the endobronchial mucosa looked scraggly like cobblestone with pink colour (Figure 2). BALF was collected from the lesions according to pulmonary CT scans and direct smear of aspirate was conducted with Ziehl-Neelsen acid-fast staining to detect tubercle bacillus. Tubercle bacillus presented under microscope (Figure 3).

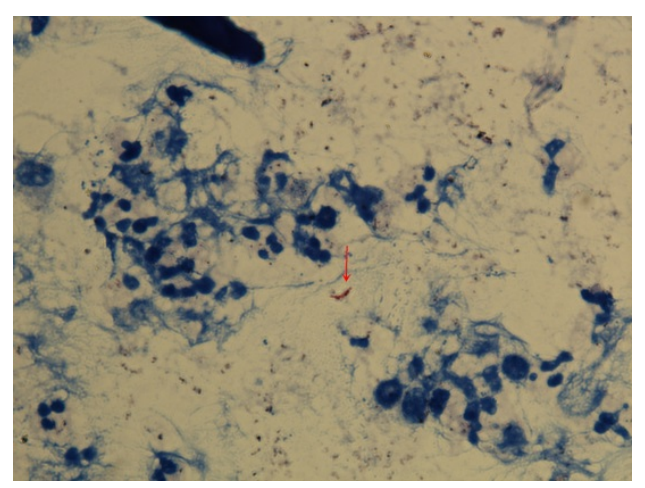

Figure 3: Acid-fast bacilli staining was positive form the sediment of BALF aspirated from the diseased region. (Amplified 100X).

\section{Case 2}

A ten years old girl who suffered from recurrent pneumonia two times within a period of six months was admitted into pediatric pulmonology. The chief complaints of the girl were fever and cough for the past twenty-one days without chest pain. The girl also suffered from hemoptysis, marasmus and night sweats. Even though her grandfather had pulmonary tuberculosis many years ago, she had no contact with her grandfather. The physical examination revealed the following: T: $37.2^{\circ} \mathrm{C}$, P: 96 beats/min, RR: 35 times/min, BP: 88/62 $\mathrm{mmHg}$, BW: $29 \mathrm{kgs}$. There were absence of rashes and enlargement of lymph nodes in jowl, axilla and groin. Liver was not palpable. No abnormalities of the heart and lungs on auscultation. Valuable accessory examinations during the time of illness were: The peripheral blood routine test illustrated that WBC was $11.4 \times 10^{9} / \mathrm{L}$, the percentage of neutrophil was $74.5 \%$, platelet was $350 \times 10^{9} / \mathrm{L}$, hemoglobin was $113 \mathrm{~g} / \mathrm{L}$, C-reactive protein (CRP) was $19.40 \mathrm{mg} / \mathrm{L}$ and procalcitonin was $0.36 \mathrm{ng} / \mathrm{mL}$. Mycoplasma pneumoniae antibody and Mycoplasma pneumoniae antibody IgM were negative. Chamydia pneumoniae antibody IgM and serum tuberculosis antibody (colloidal gold immunization) were also found to be negative. Furthermore, the tuberculin skin test (purified protein derivative, PPD) and the polymerase chain reaction (PCR) for diagnosis of tuberculosis results were negative. Cardiac enzymes were within normal range. No abnormalities of enzymes regarding the liver and kidney functions were reported. Chest contrast-enhanced CT showed that there was a consolidation with bronchiectasis in the middle lobe of the right lung (Figures 4 and 5). Lymph nodes were fused at the right pulmonary hilum. No calcification and pleural involvement and cavity formation were observed.

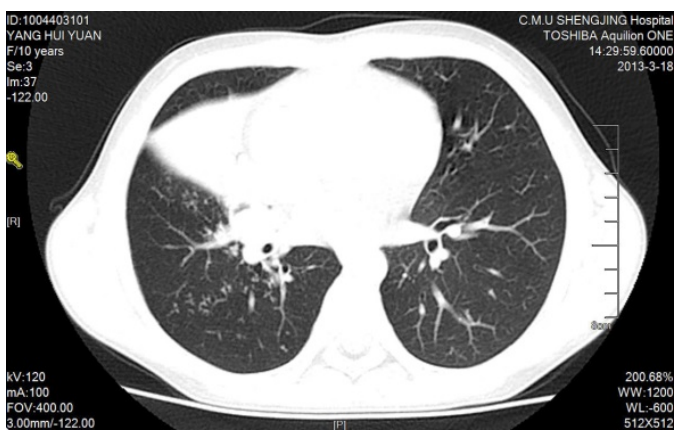

Figure 4: CT scanning of a ten years old girl with recurrent pneumonia with TBTB shows right middle lobe atelectasis.

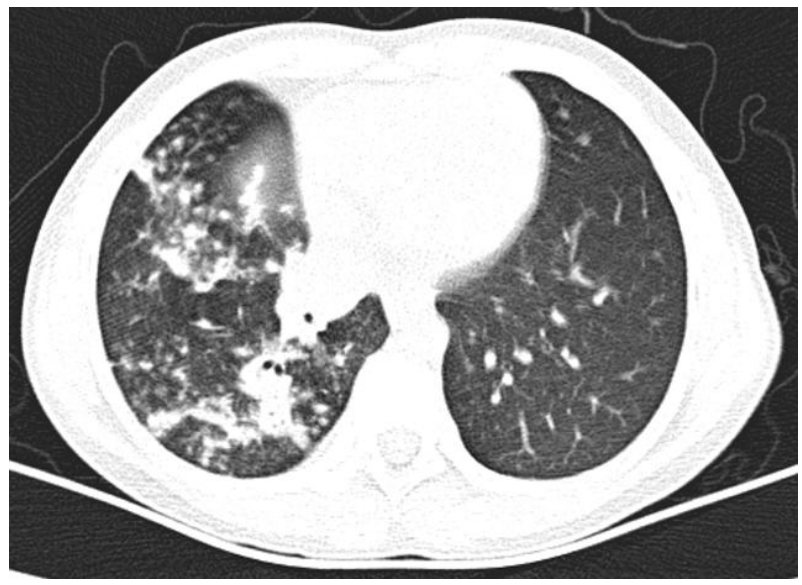

Figure 5: Computer tomography of a ten years old girl with TBTB. Nodular, plaque-like and patch-like shadows presented on the right inferior lobe with the enlargement of right lung hilus.

After nearly half month of anti-Gram positive bacteria and antiMycoplasma pneumoniae treatment, there was no improvement. So flexible bronchoscopy (Olympus BF-XP60F, $\Phi: 2.8 \mathrm{~mm}$ ) was performed under local anesthesia with the permission of her parents. The vision showed that the mucosa was bleeding and presence of erosion and adhesion with cheese-like material (Figure 6). BLAF was obtained from the right middle lobe and examined for pathogenic analysis. Biopsy was also taken from the lesion. Bleeding was stopped immediately by the local application of adrenaline. After the sedimentation, BALF smears demonstrated that acid-fast bacilli were positive (Figure 7). Both Gram-stain and $\mathrm{KOH}$ stain were negative.

\section{Case 3}

A seven years old boy had cough and intermittent fever for 40 days. The temperature waved around 38 to $39^{\circ} \mathrm{C}$. He had accepted antibiotics intravenously for 16 days at a local clinic without clear diagnosis. Since there was no signs of any improvement, cephalosporin and erythromycin were given intravenously for 10 days at local county hospital. After treatment, the temperature returned to 
Citation: Shu L, Muhammad H, Han L, Xiong Q, Hu Y, et al. (2015) The Role of Flexible Bronchoscope in the Diagnosis of the Pulmonary Tracheobronchial Tuberculosis in Children-Report of Four Cases and Review of Literature. J Bacteriol Parasitol 6: 223. doi: $10.4172 / 2155-9597.1000223$

Page 4 of 7

normal. Furthermore, azithromycin was given intravenously. The temperature recurrently soared with the same periodical features. As a result, he was administered with cefepime hydrochloride intravenously for 2 days after his referral from the affiliated hospital of Liaoning Chinese Traditional Medicine University.

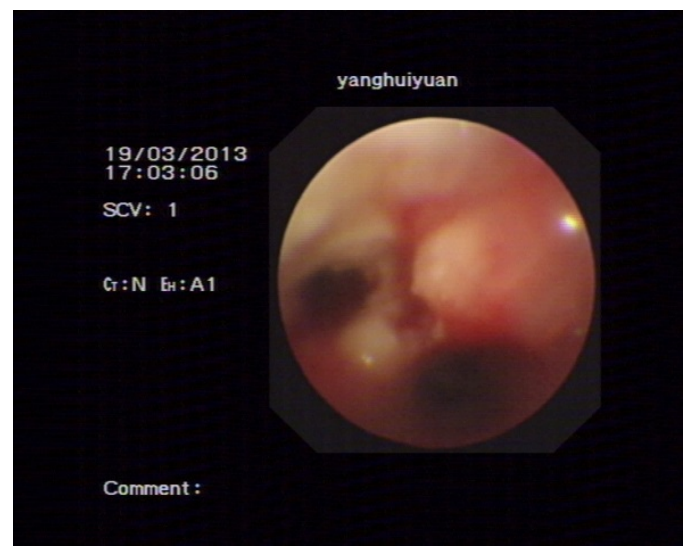

Figure 6: Bronchoscopic view seen commonly in endobronchial tuberculosis (EBTB): the actively caseating. The cheese-like material covered at the right milled and low lobes with mucosal edema and hyperemia.

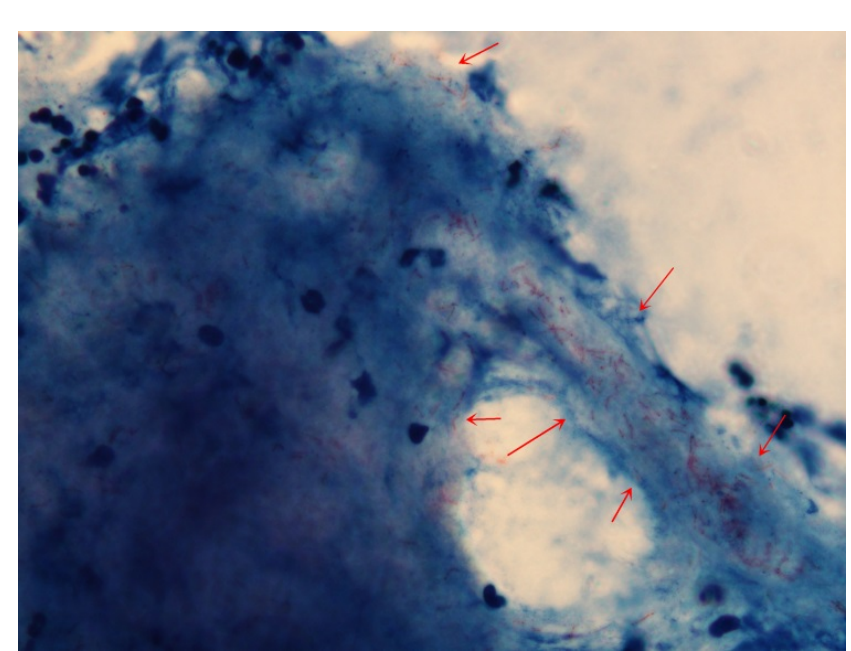

Figure 7: Great amount of acid-fast staining positive bacilli presented by the smear of BALF sediment (see arrows). (Amplified $100 \mathrm{X})$.

Then cefepime hydrochloride was given intravenously for 2 days. The boy coughed more severely and had normal temperature. There were no signs of general tiredness and night sweat after illness. His brother suffered from pulmonary tuberculosis 4 years ago. There was close connection with his brother. History of bacillus calmette-guérin (BCG) vaccine inoculation after birth was noted.

After admission, physical and laboratory tests were performed immediately. The physical examination revealed the following: $\mathrm{T}$ : $37.7^{\circ} \mathrm{C}$, P: 114 beats/min, RR: 24 times/min, BP: 90/60 mmHg, BW: 21 kgs. There was absence of rashes. Enlargement of lymph nodes in jowl, axilla, groin was not seen. Absence of moist rales and wheezing were noted on lung auscultation. Heart was also normal on auscultation. Liver was not palpable.

\section{Valuable accessory examinations during time of illness}

The peripheral blood routine test illustrated that WBC was $10.9 \times$ $10^{9} / \mathrm{L}$, the percentage of neutrophil was $72.2 \%$, platelet was $443 \times$ $10^{9} / \mathrm{L}$, hemoglobin was $111 \mathrm{~g} / \mathrm{L}$. C-reactive protein (CRP) was 62.80 $\mathrm{mg} / \mathrm{L}$. Procalcitonin was $0.26 \mathrm{ng} / \mathrm{mL}$ and Mycoplasma pneumoniae antibody was 1:320 (particle agglutination test). Mycoplasma pneumoniae antibody IgM and Chamydia pneumoniae antibody IgM were both negative. The DNA of Mycoplasma pneumoniae was also negative. The Antistreptolysin- $\mathrm{O}<25.0 \mathrm{IU} / \mathrm{mL}$. Mumps virus antibody IgM was positive. Adenovirus-IgA and parainfluenza virus-IgA were positive. Negative tuberculin skin test (purified protein derivative, PPD) was observed. Serum tuberculosis antibody (colloidal gold immunization) was weakly positive. Serum polymerase chain reaction (PCR) test for detection of tuberculosis was also negative.

\section{Disseminated intravascular coagulation (DIC)}

The following values were reported. Fibrinogen: $4.8 \mathrm{~g} / \mathrm{L}, \mathrm{D}$-dimer $220 \mu \mathrm{g} / \mathrm{L}$. Total protein value was $59.9 \mathrm{~g} / \mathrm{L}$ and albumin was $36.60 \mathrm{~g} / \mathrm{L}$. Similarly, alanine transaminase was $11 \mathrm{U} / \mathrm{L}$ and glutamic oxalacetic aminopherase was $19 \mathrm{U} / \mathrm{L}$. Creatine kinase isoenzyme-MB (CK-MB) was $26 \mathrm{U} / \mathrm{L}$ and lactate dehydrogenase (LDH) was $348 \mathrm{U} / \mathrm{L}$. Similarly, immunoglobulin A, immunoglobulin $\mathrm{G}$, and immunoglobulin $\mathrm{M}$ were $2.02 \mathrm{~g} / \mathrm{L}, 6.20 \mathrm{~g} / \mathrm{L}$ and $1.41 \mathrm{~g} / \mathrm{L}$ respectively.

Chest CT imaging showed that there was a consolidation on the right inferior lobe with cavity formation. There were no lymph node enlargement and calcification in the images. In order to make a clear diagnosis, with the permission of the parents, bronchoscopy was performed under local anesthesia after 24 days of fever. The mucosa at the inferior lobe was covered with white cheesy material (Figure 8). Biopsy was obtained from the cheese-like material. It reported the presence of inflammatory granulation tissue and local necrosis at the illness lesion (Figure 9).

BALF obtained from the right inferior lobe was centrifuged. The sedimentation was smeared immediately with ordinary Gram stain, acid-fast stain and $\mathrm{KOH}$ stain. The final result confirmed the acid-fast bacilli in BALF (Figure 10). Finally, it was diagnosed as pulmonary tuberculosis.

\section{Case 4}

A sixteen months boy had cough and wheezing accompanied for twenty-one days. The highest temperature recorded was about $40^{\circ} \mathrm{C}$. Cephalosporin was given intravenously for three days. Cough and wheezing improved as a result of it. However, two days later, the boy had vomiting and diarrhea for four days without any signs of improvement.

Another dose of cephalosporin was administered intravenously for the following 11 days after referral to the Dalian children's hospital. The boy still coughed and was wheezing. The episodes of vomiting and diarrhea were still persistent. Finally, he was transferred to our hospital. There were no signs of general tiredness and night sweat after illness. The boy had no past medical history regarding pulmonary tuberculosis. There were no other connections regarding pulmonary 
Citation: Shu L, Muhammad H, Han L, Xiong Q, Hu Y, et al. (2015) The Role of Flexible Bronchoscope in the Diagnosis of the Pulmonary Tracheobronchial Tuberculosis in Children-Report of Four Cases and Review of Literature. J Bacteriol Parasitol 6: 223. doi: $10.4172 / 2155-9597.1000223$

Page 5 of 7

tuberculosis in the case of the boy. Bacillus calmette-guerin (BCG) vaccine was inoculated after birth.

After admission, physical and laboratory tests were performed immediately. The physical examination: $\mathrm{T}: 38.3^{\circ} \mathrm{C}, \mathrm{P}: 124$ beats $/ \mathrm{min}$, RR: 25 times/min, BP: 101/65 mmHg, BW: $12 \mathrm{kgs}$. The boy was dispirited. There were no rashes present. There were no flaring of nares and three depressions sign. No enlargement of the lymph nodes in jowl, axilla and groin were seen. Moist rales and wheezing could be heard by lung auscultation. No abnormalities on heart auscultation were heard. Liver was palpable $2 \mathrm{~cm}$ under the inferior rib on the midclavicular line. There was non-pitting edema on the dorsum of the feet.

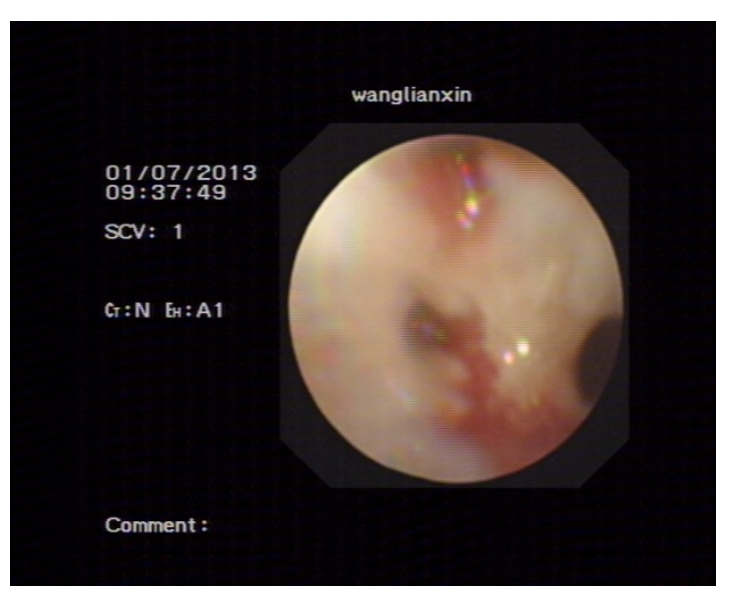

Figure 8: Bronchoscope showed the mucosa at right inferior lobe was covered by cheese-like material with mucosal edema and hyperemia. The entrance of the right inferior lobe were narrowed and occluded.

\section{Valuable accessory examinations during time of illness}

The peripheral blood routine test showed that WBC was $6.2 \times$ $10^{9} / \mathrm{L}$, the percentage of neutrophil was $58.1 \%$, platelet was $134 \times$ $10^{9} / \mathrm{L}$, hemoglobin was $94 \mathrm{~g} / \mathrm{L}$, C-reactive Protein (CRP) was $6.7 \mathrm{mg} / \mathrm{L}$ and procalcitonin was $0.82 \mathrm{ng} / \mathrm{mL}$. It was observed that the Mycoplasma pneumoniae antibody was negative (particle agglutination test) and also Mycoplasma pneumoniae antibody IgM was negative. The Chamydia pneumoniae antibody IgM was negative. Furthermore, the DNA of Mycoplasma pneumoniae was also negative and antistreptolysin-O was less than $25.0 \mathrm{IU} / \mathrm{mL}$. The mumps virusIgM and adenovirus-IgA were positive. Parainfluenza virus-IgA was positive. Tuberculin skin test (purified protein derivative, PPD) was negative. Serum tuberculosis antibody (colloidal gold immunization) and serum polymerase chain reaction (PCR) in detection of tuberculosis were also negative.

\section{Disseminated intravascular coagulation (DIC)}

Fibrinogen: $2.4 \mathrm{~g} / \mathrm{L}, \mathrm{D}$-dimer $1051 \mu \mathrm{g} / \mathrm{L}$. Total protein was $55.7 \mathrm{~g} / \mathrm{L}$, albumin was $26.3 \mathrm{~g} / \mathrm{L}$. Alanine transaminase was $17 \mathrm{U} / \mathrm{L}$. Glutamic oxalacetic aminopherase was $61 \mathrm{U} / \mathrm{L}$. Mumps virus-IgM, adenovirusIgA, and parainfluenza virus-IgA were all negative. Creatine kinase isoenzyme-MB (CK-MB) was $33 \mathrm{U} / \mathrm{L}$. Lactate dehydrogenase (LDH) was $866 \mathrm{U} / \mathrm{L}$. Immunoglobulin A was $0.117 \mathrm{~g} / \mathrm{L}$. Immunoglobulin $\mathrm{G}$ was $2.43 \mathrm{~g} / \mathrm{L}$. Immunoglobulin $\mathrm{M}$ was $0.318 \mathrm{~g} / \mathrm{L}$. 1-3- $\beta$-D-glucan $<10$ ng/L. Chest CT illustrated multiple patchy high-density lesions containing air-bronchogram, accompanied with segmental consolidation on the right lung ( Figure 11). The most common findings on CT of chest were floccus high-density lesions, part of them with aerial bronchogram, and segmental consolidation on the right lung.

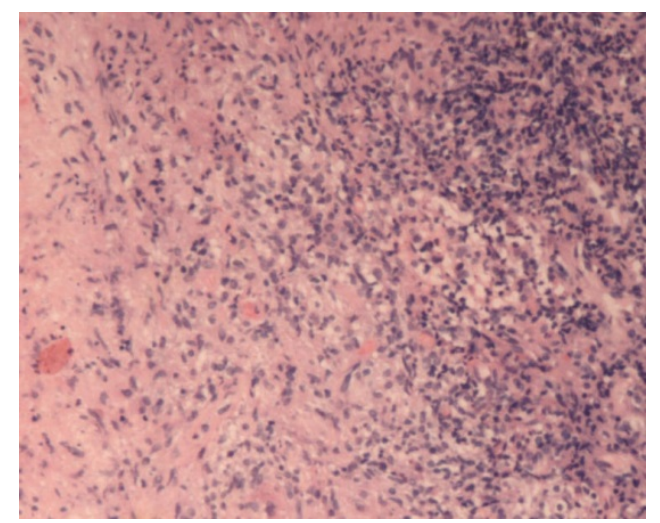

Figure 9: Inflammatory granuloma, local necrosis presented at diseased region.

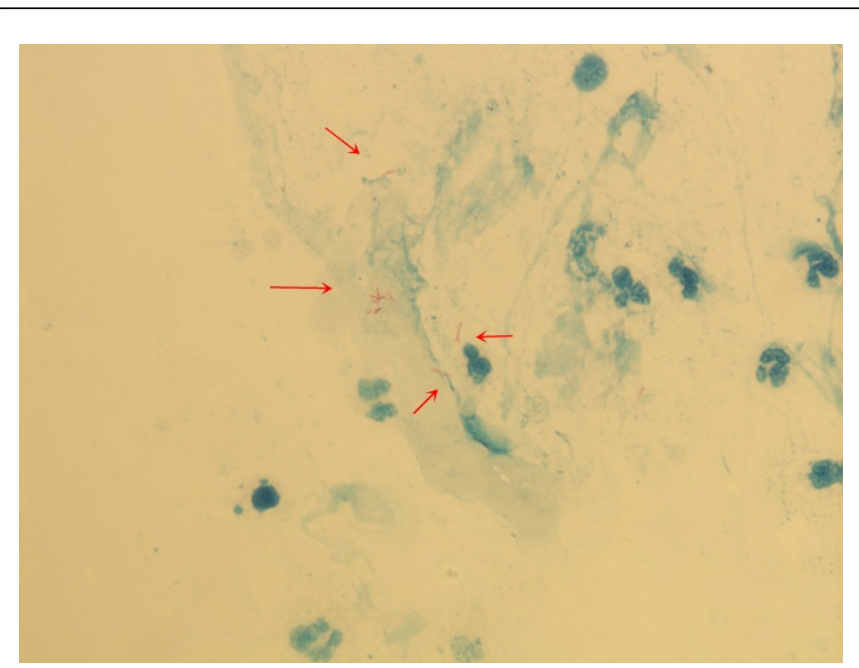

Figure 10: Acid-fast staining positive bacilli scattered on the slices of BALF aspirates (see arrows). (Amplified 100X).

Corticosteroids were given intravenously for the treatment of wheezing and fever. As a result, the temperature decreased for 5 days. However, the temperature increased again. The child was sleepy and more irritable without stiff neck and other meningeal irritation sign (Kernig and Brudzinski signs were negative). Lumbar puncture was performed immediately after local anesthesia in order to exclude intracranial infection. It showed that the velocity of cerebral spinal fluid was about 60 drops per minute. Cellular analysis and biochemical tests were normal. Gram stain, acid-fast stain and $\mathrm{KOH}$ stain of cerebral spinal fluid were reported as negative. Maxipime, azithromycin, and adenine arabinoside monophosphate were given intravenously every day for one week, accompanied with oral 
Citation: Shu L, Muhammad H, Han L, Xiong Q, Hu Y, et al. (2015) The Role of Flexible Bronchoscope in the Diagnosis of the Pulmonary Tracheobronchial Tuberculosis in Children-Report of Four Cases and Review of Literature. J Bacteriol Parasitol 6: 223. doi: $10.4172 / 2155-9597.1000223$

Page 6 of 7

administration (administer) of fluconazole capsule. The temperature was still very high.

Therefore, after the consent from the medical ethics committee in our hospital and the parents were attained, Bronchoscopy was performed under local anesthesia. The mucosa was pink and smooth without erosion and cheese-like substances (Figure 12). No mucosa abnormalities were observed under electronic flexible bronchoscope. Acid-fast positive bacilli were found under microscope with acid-fast stain (Figure 13). Pulmonary tuberculosis was diagnosed with the help of bronchoscopy, even though there were no TB contacting history and typical TB manifestations.

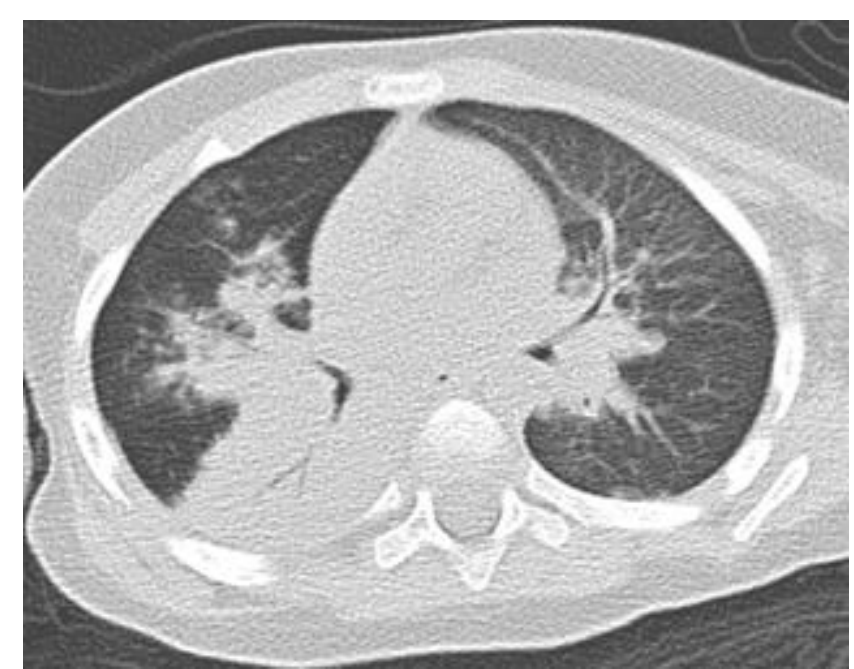

Figure 11: Computer tomography of a sixteen months boy with TBTB. Enlargement of right and left hilus around with large area of shadow. Consolidation occurred in the right inferior lobe with airbronchogram in it.

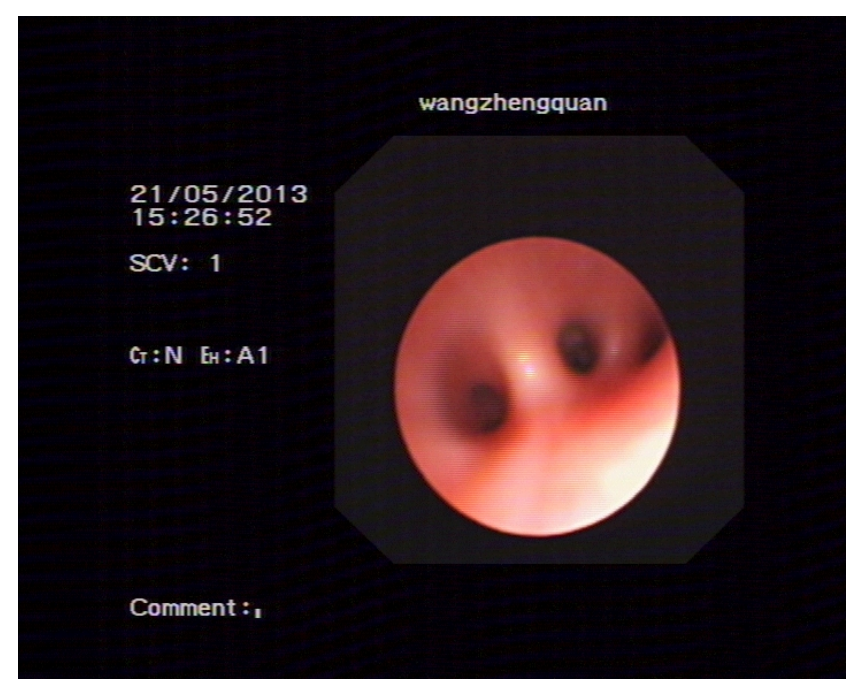

Figure 12: A special appearance of TBTB with no pathological changes grossly. It was regarded as the beginning of Mycobacterium tuberculosis infection.

\section{Discussion}

A number of studies have confirmed the usefulness of flexible bronchoscopy in the diagnosis of pulmonary tuberculosis [9-11]. The use of flexible fiberoptic bronchoscopy with bronchial aspiration and bronchial alveolar fluid lavage under local anesthesia is a relatively safe procedure and well tolerated by most of the pediatric patients. The diagnostic yield and the safety of the procedure have been documented in earlier studies. There are known complications but occur rarely [2].

The diagnosis of tuberculosis in children is complicated and difficult. The first reason is that it commonly relies on clinical signs and symptoms, tuberculin skin testing and chest radiograph. The clinical symptoms are nonspecific. Furthermore, the tuberculin skin test (PPD) and chest radiographs fail to present unique feature demonstrated only by $\mathrm{TB}$, and also the routine laboratory tests are not valuable. There are two situations, which lead the clinician to suspect that a child has tuberculosis. The first scenario (a) is when there is a history of chronic illness with clear symptoms such as cough and/or fever, weight loss or failure to thrive, an inability to return to normal health after measles or whooping cough, fatigue, and wheezing. The second scenario (b) is when there is one or more of the following signs: malnutrition, lymphadenopathy, chest signs, hepatomegaly and/or splenomegaly, meningeal signs, and/or ascites. From case one report, you can see that the pulmonary tuberculosis in childhood was often confused with refractory and/or complicated pneumonia, even though all tests on TB had been performed. It implied that the repeated pneumonia with gigantic splenomegaly, and poor response to two weeks antibiotic treatment might lead the pediatrician to suspect that a child maybe have tuberculosis. It is necessary to perform a bronchoscopy for differential diagnosis [13].

Symptoms of childhood TB are nonspecific, and up to $50 \%$ of children may be asymptomatic in early stages of the disease. So far, there is the absence of a practical reference test or gold standard $[14,15]$. For the rest three cases, more rapid and sensitive laboratory tests in molecular biology and immunology have been employed for diagnosis $[16,17]$ but the results for children have been disappointing.

Better techniques would immensely benefit children and infants in whom early diagnosis is imperative for preventing progressive $\mathrm{TB}$ $[2,18]$. A number of studies have confirmed the usefulness of fiberoptic fronchoscopy in the diagnosis of pulmonary tuberculosis [9]. In Cakir's study [6], 33 (47\%) cases among 70 pediatric patients were identified as cases of endobronchial tuberculosis (EBTB).

Flexible bronchoscopy (FB) is the necessary method for the diagnosis of tracheobronchial tuberculosis (TBTB). FB offers a safe and rapid means of confirming the diagnosis by direct visualization of the airway abnormalities [6]. The advantage from FB includes vast information such as types of TBTB, site, range and severity of illness, as well as the state of stenosis, obstruction and softness of the bronchi. Transbronchial biopsy, brush smear on the diseased region, and acidfast bacilli staining of BALF sediment can provide the pathological evidence for the diagnosis of TBTB [19].

Pathological changes typically contain congestion of mucosa, edema, hyperplasia, erosion, ulcer, necrosis, granuloma, scar, and bronchiolymphatic fistula of bronchus under bronchoscope. Histopathological manifestations include exudation, proliferation and denaturalization. Presentation of epithelioid cell, Langhans' giant cell, caseation can be helpful for the diagnosis of TB [4]. 
Citation: Shu L, Muhammad H, Han L, Xiong Q, Hu Y, et al. (2015) The Role of Flexible Bronchoscope in the Diagnosis of the Pulmonary Tracheobronchial Tuberculosis in Children-Report of Four Cases and Review of Literature. J Bacteriol Parasitol 6: 223. doi: $10.4172 / 2155-9597.1000223$

Page 7 of 7

In our cases, caseation often covered the illness lesions, accompanied with mucosa congestion, exudation, bleeding and erosion (Figures 6 and 8). It implies that the bronchial involvement was frequent in childhood TB [6]. Sometimes it was confused with the presentations of Mycoplasma penumoniae infection or fungal infection (such as Aspergillus fumigatus) under the bronchoscope.

Early detection and effective treatment of ETB are important to decrease secondary complications, such as bronchiectasis and bronchial stenosis [6]. In rare case, there was no abnormality under bronchoscope (Figure 12). It might be inferred that mucosa changes does not occur in the early stage. As a result, it is not enough to make a definite diagnosis and differential diagnosis based on the manifestations of mucosa under bronchoscope. Under this condition, it really depends on bacteriological detection.

The study of BALF can be valuable for the diagnosis of TB. The percentage of lymphocytes in BALF may be increased in TB and can be confirmed by cytological analysis. But it is not unique to confirm the diagnosis of tuberculosis because it will increase in some other conditions such as pediatric lymphocytic interstitial pneumonia (LIP).

For the confirmation of TB, it is valuable and important to conduct pathogenic examination from BALF. Aspirations from these four cases were smeared for acid-fast bacilli with Ziehl-Neelsen technique and showed positive results (Figure 13). Aspirates obtained from bronchoscopy, is superior to gastric washings because of the higher positive rate. It should be should be regarded as optimum for the diagnosis of sputum and/or gastric washings smear-negative pulmonary TB $[9,20,21]$.

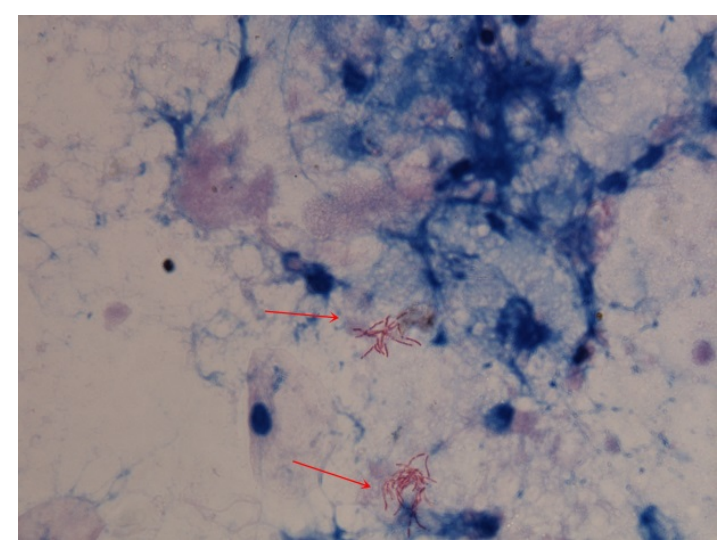

Figure 13: Acid-fast staining positive bacilli presented on the smear of BALF sediment (see arrows). (Amplified 100X).

In conclusion, it is difficulty for pediatrician to make a confirmed diagnosis of childhood TB on clinical and radiological grounds in early stage or differential diagnosis from refractory pneumonia. Flexible bronchoscopy provides pathological and etiological confirmation for diagnosis of tuberculosis with great safety and efficiency as well as intuition. It is useful for diagnosis and differential diagnosis of pulmonary tuberculosis from complicated lung infections.

\section{References}

1. Dye C, Scheele S, Dolin P, Pathania V, Raviglione MC (1999) Consensus statement. Global burden of tuberculosis: estimated incidence, prevalence, and mortality by country. WHO Global Surveillance and Monitoring Project. JAMA 282: 677-686.

2. Ávalos GGL, Montes de Oca EP (2012) Classic and new diagnostic approaches to childhood tuberculosis. J Trop Med 2012: 818219.

3. World Health Organization (2006) Guidance for national tuberculosis programmes on management of tuberculosis in children. (2ndedn), Geneva.

4. Xue Q, Wang N, Xue X, Wang J (2011) Endobronchial tuberculosis: an overview. Eur J Clin Microbiol Infect Dis 30: 1039-1044.

5. Lee JH, Park SS, Lee DH, Shin DH, Yang SC, et al. (1992) Endobronchial tuberculosis. Clinical and bronchoscopic features in 121 cases. Chest 102: 990-994.

6. Cakir E, Uyan ZS, Oktem S, Karakoc F, Ersu R, et al. (2008) Flexible bronchoscopy for diagnosis and follow up of childhood endobronchial tuberculosis. Pediatr Infect Dis J 27: 783-787.

7. Qingliang X, Jianxin W (2010) Investigation of endobronchial tuberculosis diagnoses in 22 cases. Eur J Med Res 15: 309-313.

8. Uzuner N, Anal O, Karaman O, Sevinç C, Türkmen M, et al. (2003) Endobronchial tuberculosis complicated with Staphylococcus aureus pneumonia and empyema in a child. Turk J Pediatr 45: 254-257.

9. Quaiser S, Agarwal A, Khan R, Haque SF (2012) Fiberoptic bronchoscopy, as a valuable diagnostic option in sputum negative pulmonary tuberculosis: A prospective study. Int J Appl Basic Med Res 2: 123-127.

10. Chan S, Abadco DL, Steiner P (1994) Role of flexible fiberoptic bronchoscopy in the diagnosis of childhood endobronchial tuberculosis. Pediatr Infect Dis J 13: 506-509.

11. García AT, Gómez de Agüero MIB, Carrasco CM, Landeira CA, Dorado RD, et al. (2004) Fiberoptic bronchoscopy in childhood endobronchial tuberculosis. An Pediatr (Barc) 61: 314-319.

12. Kalawat U, Sharma KK, Reddy PN, Kumar AG (2010) Study of bronchoalveolar lavage in clinically and radiologically suspected cases of pulmonary tuberculosis. Lung India 27: 122-124.

13. Charoenratanakul S, Dejsomritrutai W, Chaiprasert A (1995) Diagnostic role of fiberoptic bronchoscopy in suspected smear negative pulmonary tuberculosis. Respir Med 89: 621-623.

14. Marais BJ, Gie RP, Hesseling AC, Schaaf HS, Lombard C, et al. (2006) A refined symptom-based approach to diagnose pulmonary tuberculosis in children. Pediatrics 118: e1350-1359.

15. Marais BJ, Pai M (2007) New approaches and emerging technologies in the diagnosis of childhood tuberculosis. Paediatr Respir Rev 8: 124-133.

16. Zar HJ, Connell TG, Nicol M (2010) Diagnosis of pulmonary tuberculosis in children: new advances. Expert Rev Anti Infect Ther 8: 277-288.

17. Coulter JB (2008) Diagnosis of pulmonary tuberculosis in young children. Ann Trop Paediatr 28: 3-12.

18. Khan EA, Starke JR (1995) Diagnosis of tuberculosis in children: increased need for better methods. Emerg Infect Dis 1: 115-123.

19. Charoenratanakul S, Dejsomritrutai W, Chaiprasert A (1995) Diagnostic role of fiberoptic bronchoscopy in suspected smear negative pulmonary tuberculosis. Respir Med 89: 621-623.

20. Dickson SJ, Brent A, Davidson RN, Wall R (2003) Comparison of bronchoscopy and gastric washings in the investigation of smearnegative pulmonary tuberculosis. Clin Infect Dis 37: 1649-1653.

21. Schoch OD, Rieder P, Tueller C, Altpeter E, Zellweger JP, et al. (2007) Diagnostic yield of sputum, induced sputum, and bronchoscopy after radiologic tuberculosis screening. Am J Respir Crit Care Med 175: 80-86. 